\title{
Graviatoms with de Sitter Interior
}

\author{
Irina Dymnikova ${ }^{1,2}$ and Michael Fil'chenkov ${ }^{3}$ \\ ${ }^{1}$ Department of Mathematics and Computer Science, University of Warmia and Mazury, Słoneczna 54, 10-710 Olsztyn, Poland \\ ${ }^{2}$ A. F. Ioffe Physico-Technical Institute, Politekhnicheskaja 26, St. Petersburg 194021, Russia \\ ${ }^{3}$ Institute of Gravitation and Cosmology, Peoples' Friendship University of Russia, 6 Miklukho-Maklay Street, Moscow 117198, Russia \\ Correspondence should be addressed to Irina Dymnikova; irina@uwm.edu.pl
}

Received 25 July 2013; Accepted 18 September 2013

Academic Editor: Maxim Khlopov

Copyright (C) 2013 I. Dymnikova and M. Fil'chenkov. This is an open access article distributed under the Creative Commons Attribution License, which permits unrestricted use, distribution, and reproduction in any medium, provided the original work is properly cited.

\begin{abstract}
We present a graviatom with de Sitter interior as a new candidate to atomic dark matter generically related to a vacuum dark energy through its de Sitter vacuum interior. It is a gravitationally bound quantum system consisting of a nucleus represented by a regular primordial black hole (RPBH), its remnant or gravitational vacuum soliton G-lump, and a charged particle. We estimate probability of formation of RPBHs and G-lumps in the early Universe and evaluate energy spectrum and electromagnetic radiation of graviatom which can in principle bear information about a fundamental symmetry scale responsible for de Sitter interior and serve as its observational signatures.
\end{abstract}

\section{Introduction}

Nonluminous atomic dark matter includes a wide range of candidates starting from the historically first mirror dark matter [1-3]. Presented here is graviatom, a gravitationally bound quantum system consisting of a regular black hole or gravitational soliton G-lump and a captured charged particle, which can be formally classified as atomic dark matter with an additional intrinsic dark feature presented by dark energy interior of a certain fundamental scale of de Sitter vacuum. Electromagnetic radiation of a graviatom bearing information on this scale fits in the ultrahigh gamma range and can be regarded as its independent observational signature.

The idea of an atom with a black hole as a nucleus goes back to 1971 when Hawking put forward the idea that primordial charged black holes could capture free charged particle forming neutral and nonrelativistic ultraheavy black hole atoms [4]. The minimal mass of a primordial black hole that would not have evaporated entirely by now is $M \sim$ $5 \times 10^{14} \mathrm{~g}$ [5]. The term gravitational atom was introduced in [6] for gravitationally bound neutral black hole and a charged particle. Similar quantum system called a hypothesized gravitational atom was considered in [7] by solving the Schrödinger equation with the Newtonian gravitational potential of a point-like mass modified by the presence of extra compactified dimensions and a captured neutron [7].

Quantum levels in the field of a black hole have been studied in detail starting from 1987 [8-10]. The gravitational analogue of the hydrogen atom orbitals was considered in [11] where the spectrum of normalizable fermion bound states was calculated in the Schwarzschild background. Perturbative modification of electronic orbitals around charged black holes was proposed in [12]. It is clear that a particle undergoing transitions between quantum levels should radiate. However, this question has been much less studied in the literature than the question of quantum spectrum of a black hole.

Quantum radiation of a charged nonrelativistic particle in the Schwarzschild field was first studied in 1990 in [13] with taking into account the DeWitt conservative self-force whose origin is interaction of an electrostatic field of a charged particle with a gravitational field of a source [14]. It was shown that in this case the DeWitt self-force always exceeds the nonconservative radiation damping force; the energy spectrum and intensity of the electric dipole radiation were calculated for the case of black hole with masses ranging from $10^{-5} \mathrm{~g}$ to $10^{14} \mathrm{~g}$ [13] (see also [15]). The conditions of existence 
of a gravitationally bound quantum system with a neutral black hole as a nucleus were formulated in $[16,17]$ where it was called graviatom.

In this paper we study graviatoms with regular black holes with de Sitter interior [18], their remnants [19, 20], and vacuum gravitational solitons G-lumps $[19,21]$ as nuclei.

Primordial black hole remnants left after the Hawking evaporation have been considered as a source of dark matter for more than two decades [22-29] (for a review see [30$32])$. They could also be a source of additional information about the primordial power spectrum $[29,33,34]$ and about their effect on big bang nucleosynthesis $[29,35]$. However, the question whether some remnants leave after the Hawking evaporation of a singular black hole still remains open. The generalized uncertainty principle requires existence of a black hole remnant as a Planck size black hole [25]. On the other hand, no evident symmetry or quantum number was found which would prevent a complete evaporation [36]. Another question in the case of a singular black hole is how to evaporate a singularity? The complete evaporation would involve a serious and unclear change in spacetime structure which would have to evolve from a singularity to a maximally symmetric Minkowski (or de Sitter) space [37].

These problems do not arise in the case of a regular black hole with de Sitter centre instead of a singularity which leaves behind a thermodynamically stable double-horizon remnant [20] and does not involve a dramatic change in spacetime structure [37].

The idea of de Sitter interior goes back to the heuristic hypothesis that de Sitter vacuum could be a final state in a collapse [38], to the idea of a self-regulatory behavior of space-time geometry at achieving the Planckian densities [39], and to the idea of the existence of the limiting curvature of the Planck scale [40]. Second-order phase transition during evaporation of a black hole with de Sitter interior $[19,20]$ suggests possibility for a phase transition to de Sitter vacuum in the origin [41].

Arguments in favour of a regular black hole are provided by a loop quantum gravity $[42,43]$. The "renormalization group improving" approach based on the running Newton constant, applied to the Schwarzschild spacetime, predicts an appearance of a smooth de Sitter core replacing a singularity [44]. The noncommutative geometry approach (for a review see [45]) applied to the Schwarzschild black hole leads to a regular de Sitter core at short distances from the origin [46]. Appearance of de Sitter core was found also for a cosmological noncommutative black hole of positive mass with the Gaussian density profile [47].

The Einstein equations admit the class of regular spherically symmetric solutions asymptotically de Sitter as $r \rightarrow 0$ with $\Lambda=8 \pi G \rho_{\text {int }}, \rho_{\text {int }}=\rho(r \rightarrow 0)$ of a certain fundamental scale and at infinity $[21,48,49]$. A source term for this class is specified by $T_{0}^{0}=T_{1}^{1}$ and provides a unified modelindependent description of dark ingredients in the Universe by a vacuum dark fluid $[50,51]$. In this approach a vacuum dark energy is described by a time evolving and spatially inhomogeneous cosmological term [48], while dark matter is represented by compact objects generically related to vacuum dark energy through de Sitter vacuum interior. They include

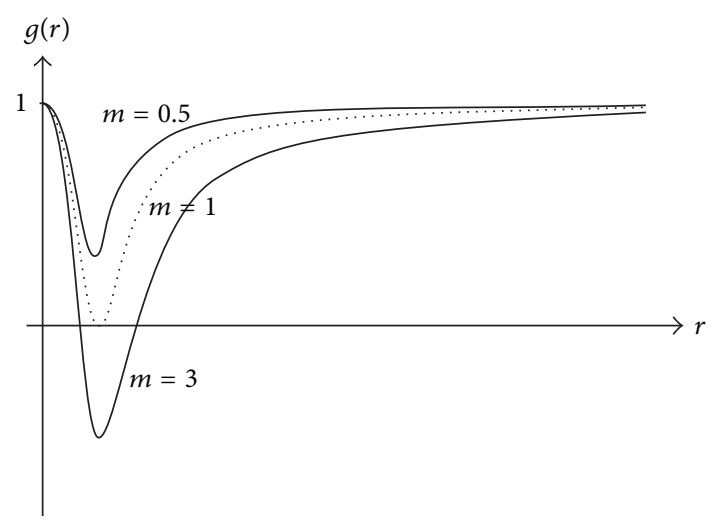

FIgure 1: The metric function $g(r)$ for de Sitter-Schwarzschild spacetime; $m=M / M_{\text {crit }}$.

regular black holes $[18,52,53]$, their remnants [20], and gravitationally bound vacuum structures without black hole horizon (dark particles or dark stars, dependently on a mass) $[19,21]$, called G-lumps, since they hold themselves together by their own gravity [21]. For this class a static metric is described by the line element:

$$
d s^{2}=g(r) d t^{2}-\frac{d r^{2}}{g(r)}-r^{2} d \Omega^{2} .
$$

In the asymptotically flat case to be studied in this paper, the metric function reads

$$
\begin{gathered}
g(r)=1-\frac{\mathscr{R}_{g}(r)}{r}, \quad \mathscr{R}_{g}(r)=2 G \mathscr{M}(r), \\
\mathscr{M}(r)=4 \pi \int_{0}^{r} \rho(x) x^{2} d x .
\end{gathered}
$$

A metric function $g(r)$ is asymptotically Schwarzschild at $r \gg r_{*}$, where $r_{*}=\left(r_{\text {int }}^{2} r_{g}\right)^{1 / 3}$ is the characteristic length of de Sitter-Schwarzschild spacetime [18, 19], $r_{g}=2 G M c^{-2}$, and $r_{\text {int }}$ is characteristic scale for the interior de Sitter vacuum related to its density by $r_{\text {int }}^{2}=3 c^{2} / 8 \pi G \rho_{\text {int }}$. (Characteristic length scales essential for graviatom are summarized in the Appendix.) The scale $r_{*}$ comes as the radius of the direct matching of the de Sitter and Schwarzschild metrics, $g_{\mathrm{deS}}=$ $g_{\text {Schw }}$; that is, $1-r_{*}^{2} / r_{\text {int }}^{2}=1-r_{g} / r_{*}$ [39]. It is also the scale at which the scalar curvature $R$ changes sign [19]. The mass $M=4 \pi \int_{0}^{\infty} \rho(r) r^{2} d r$ is related to interior de Sitter vacuum and to breaking of spacetime symmetry from the de Sitter group at $r=0[21,54,55]$.

Regular black hole with de Sitter centre has two horizons $[19,21]$ which coalesce in the course of evaporation at a certain mass $M_{\text {cr }}$ corresponding to a thermodynamically stable double-horizon remnant [19-21, 46, 56, 57]. For $M<M_{\mathrm{cr}}$, the metric (1)-(2) describe G-lumps. The typical behaviour of the metric function [21] is shown in Figure 1.

Primordial black holes can be formed by various mechanisms (for a review see $[35,58]$ ). Most general possibility involves primordial density inhomogeneities forming overdense regions which can stop expanding and collapse $[4,59]$. 
The particular origin of inhomogeneities can be quantum fluctuations arising in various inflationary scenarios [35]. $\mathrm{PBH}$ formation would be enhanced if inflation is followed by a dust-like stage dominated by nonrelativistic particles [58]. This point is of special interest in the context of graviatoms. As we will see below, the case of graviatom with $\mathrm{RPBH}$ remnant or G-lump and captured GUT particles, $M \sim 10^{14}$ $10^{16} \mathrm{GeV}$, which are considered as superheavy dark matter, is the most promising [60-64].

In the literature two mechanisms of producing such particles have been considered. One is gravitational production towards the end of inflation $[60-62,65,66]$. The other is decay of the inflationary de Sitter vacuum during symmetrybreaking phase transitions at the GUT scale: statistical mechanics approach with model-independent description of cosmological background shows that appearance of GUT particles from the GUT scale de Sitter vacuum looks like evaporation of Bose-condensate: particles satisfy the equation of state of an ideal quantum degenerate Bose gas with the Gibbons-Hawking temperature related to the de Sitter Horizon [67-71]. GUT particles could be captured by RPBH remnants or G-lumps and survive to the present epoch as constituents of graviatoms.

The main goal of this paper is to study typical features of graviatoms radiation which could result in their observational signatures as dark matter candidates.

In Section 2 we estimate the probability of formation of a RPBH and G-lump in a quantum collapse of a primordial fluctuation. In Section 3 we introduce the Schrödinger equation for graviatom and study typical behaviour of its potential. Section 4 is devoted to quantum spectra and mechanisms of graviatom radiation. In Section 5 we summarize the results.

\section{Formation of the Regular Primordial Black Holes and G-Lumps with de Sitter Interior}

In the classical approach geometry of a collapsing body in the comoving coordinates can be presented by the FRW spacetime with the positive curvature [72]. In quantum cosmology the wave function is defined on a superspace which is the space of all 3-dimensional geometries $g_{i j}(\vec{x})$ and matter field configuration $\varphi_{m}(\vec{x}), \Psi=\Psi\left[g_{i j}(\vec{x}), \varphi_{m}(\vec{x})\right]$. It satisfies the Wheeler-DeWitt equation $\widehat{H} \Psi=0$. In the case of the FRW cosmology with the only dynamical variable, the scale factor $a(t)$, the Wheeler-DeWitt equation reduces to the simple case called minisuperspace model. For the Friedmann closed model it reads [73]

$$
\frac{d^{2} \Psi}{d a^{2}}-V(a) \Psi=0
$$

where

$$
V(a)=\frac{1}{l_{p l}^{4}}\left(k a^{2}-\frac{8 \pi G}{3 c^{4}} \varepsilon a^{4}\right), \quad k=1
$$

In general case a collapsing quantum fluctuation can be described as a superposition of partial energy densities of possible matter contributions [74]:

$$
\varepsilon=\varepsilon_{p l} \sum_{0}^{6} A_{n}\left(\frac{l_{p l}}{a}\right)^{n} .
$$

The parameter $n$ is related to the parameter $w$ in the equation of state $p=w \varepsilon$, as $n=3(1+w)$. Contributions to density (5) include an inflationary vacuum $(n=0)$, ensembles of domain walls $(n=1)$ and strings $(n=2)$, nonrelativistic matter (dust with $n=3)$, ultrarelativistic gas $(n=4)$, perfect gas $(n=5)$, and ultrastiff matter $(n=6)$.

The Wheeler-DeWitt equation (3) reduces to

$$
-\frac{\hbar^{2}}{2 m_{p l}} \frac{d^{2} \Psi}{d a^{2}}+(U(a)-E) \Psi=0,
$$

where the potential $U(a)$ expressed in terms of the dimensionless variable $\gamma \equiv a / l_{p l}$ has the form [75]

$$
U(\gamma)=\frac{E_{p l}}{2}\left(k \gamma^{2}-B_{0} \gamma^{4}-B_{1} \gamma^{3}-B_{2} \gamma^{2}-B_{3} \gamma-\frac{B_{5}}{\gamma}-\frac{B_{6}}{\gamma^{2}}\right) .
$$

Energy of a fluctuation in (6) is

$$
E=\frac{E_{p l}}{2} B_{4} \text {. }
$$

Coefficients $B_{n}$ are related to the coefficients $A_{n}$ in (5) by $B_{n}=$ $\left(8 \pi G / 3 c^{2}\right) A_{n}$.

To model a collapse to a compact object with the de Sitter interior, we modify the potential (7) by introducing a cutoff related to characteristic scale for an interior de Sitter vacuum $\gamma_{\text {int }}$ in the terms of the negative powers of $\gamma$ corresponding to high density contributions to (5), in accordance with the underlying hypothesis [38-40] that transition to interior de Sitter vacuum may occur at achieving a certain high density during contraction. Modified potential has the form

$$
\begin{aligned}
& U(\gamma)=\frac{E_{p l}}{2}\left(k \gamma^{2}-B_{0} \gamma^{4}-B_{1} \gamma^{3}-B_{2} \gamma^{2}\right. \\
& \left.\quad-B_{3} \gamma-\frac{B_{5}}{\left(\gamma-\gamma_{\text {int }}\right)}-\frac{B_{6}}{\left(\gamma-\gamma_{\text {int }}\right)^{2}}\right) .
\end{aligned}
$$

The potential (9) tends to minus infinity for $\gamma \rightarrow \infty$ and $\gamma \rightarrow \gamma_{\text {int }}$. Near $\gamma>\gamma_{\text {int }}$, the potential (9) has a maximum $U_{m}>0$, and the process of a quantum collapse involves tunnelling through the potential barrier.

For big values of the variable $\gamma$, in the limit $\gamma \gg\left|B_{4}\right|^{1 / 4}$, the Schrödinger equation (6) reduces to

$$
\frac{d^{2} \Psi}{d \gamma^{2}}+B_{0} \gamma^{4} \Psi=0
$$

which has the solution [76]:

$$
\Psi=\sqrt{\gamma} Z_{1 / 6}\left(\frac{\sqrt{B_{0}}}{3} \gamma^{3}\right),
$$


with the asymptotics [75]:

$$
\Psi=C_{1} \exp \left(\frac{i B_{0} \gamma^{3}}{3}\right)+C_{2} \exp \left(-\frac{i B_{0} \gamma^{3}}{3}\right) .
$$

Choosing integration constant proper for a collapse, we get the $\mathrm{WKB}$ wave function corresponding to deflation. Indeed, in the limits $B_{0} \gamma^{4} \gg B_{4}$ and $\gamma \gg \gamma_{\text {int }}$, the action $S=\int L d a \propto$ $\pm a^{3}$, where $L=(d a / d \eta)^{2}+H^{2} a^{4} / c^{2}-k a^{2}, a d \eta=c d t$, and $H= \pm \dot{a} / a[75]$. The classical solutions are $a \propto e^{H t}$ (inflation) and $a \propto e^{-H t}$ (deflation).

For $\gamma$ very close to $\gamma_{\text {int }}, 0<\gamma-\gamma_{\text {int }} \ll \sqrt{B_{6} / B_{4}}$, the Schrödinger equation takes the form

$$
\frac{d^{2} \Psi}{d \gamma^{2}}+\frac{B_{6}}{\left(\gamma-\gamma_{\text {int }}\right)^{2}} \Psi=0 .
$$

Its solution, satisfying the boundary condition $\Psi\left(\gamma_{\text {int }}\right)=0$, is given by

$$
\begin{array}{r}
\Psi=\sqrt{\gamma-\gamma_{\text {int }}}\left(C_{1}\left(\gamma-\gamma_{\text {int }}\right)^{b}+C_{2}\left(\gamma-\gamma_{\text {int }}\right)^{-b}\right), \\
b^{2}=\frac{1}{4}-B_{6}>0 .
\end{array}
$$

After tunnelling through the barrier, a collapsing object appears in the potential well. In this region, for $\gamma-\gamma_{\text {int }} \ll 1$ the Schrödinger equation (6) reduces to [75]

$$
\frac{d^{2} \Psi}{d \gamma^{2}}+\left(B_{0} \gamma_{\text {int }}^{4}+\frac{B_{5}}{\left(\gamma-\gamma_{\text {int }}\right)}+\frac{B_{6}}{\left(\gamma-\gamma_{\text {int }}\right)^{2}}+\frac{2 E}{E_{p l}}\right) \Psi=0,
$$

whose solution, satisfying the boundary condition $\Psi\left(\gamma_{\text {int }}\right)=$ 0 , reads

$$
\Psi=C \rho^{s+1} e^{-\rho / 2} F(-p, 2 s+2, \rho),
$$

where $F(-p, 2 s+2, \rho)$ is the degenerate hypergeometric function, $s=-(1 / 2)+\sqrt{(1 / 4)-B_{6}}, \rho=2(\gamma-$ $\left.\gamma_{\text {int }}\right) \sqrt{-B_{0} \gamma_{\text {int }}^{4}-2 E / E_{p l}}, p=n-s-1, n=B_{5} /$ $\sqrt{-B_{0} \gamma_{\text {int }}^{4}-2 E / E_{p l}}$, and $p=0,1,2, \ldots$.

The energy spectrum is given by

$$
E_{p}=-\frac{B_{5}^{2}}{8\left(p+1 / 2+\sqrt{1 / 4-B_{6}}\right)^{2}} E_{p l}-\frac{1}{2} B_{0} \gamma_{\mathrm{int}}^{4} E_{p l} \text {. }
$$

The discrete spectrum does exist only if the following condition is satisfied:

$$
\frac{2|E|}{E_{p l}}=\left|B_{4}\right| \geq B_{0} \gamma_{\text {int }}^{4} .
$$

This allows one to estimate the probability of tunnelling through the barrier and range of masses $M$ of a collapsing object. The tunnelling probability is determined by the WKB penetration factor:

$$
D=\exp \left(-\frac{2 l_{p l}}{\hbar} \int_{\gamma_{1}}^{\gamma_{2}} \sqrt{2 m_{p l}(U-E)} d \gamma\right)=\exp (-2 \Gamma),
$$

where $\gamma_{1} \approx\left(\left|B_{4}\right| / B_{0}\right)^{1 / 4}, \gamma_{2} \approx \gamma_{\text {int }}+\sqrt{B_{6} /\left(\left|B_{4}\right|-B_{0} \gamma_{\text {int }}^{4}\right)}$, and $\gamma_{2}<\gamma_{1}$.

The factor $\Gamma$ is estimated as $\Gamma<2\left|B_{4}\right|^{3 / 4} B_{0}^{-1 / 4}$, which gives $D>\exp \left(-4\left|B_{4}\right|^{3 / 4} B_{0}^{-1 / 4}\right)$.

The Schrödinger equation (6) describes the planckeon on the energy level given by (17); $E=-\left(\left|B_{4}\right| / 2\right) E_{p l}$. Its total energy includes the rest mass, $M c^{2}+E=E_{p l}$, so the mass of an object is given by $M / m_{p l}=1+\left|B_{4}\right| / 2$. Taking into account $\left|B_{4}\right|<M / m_{p l}$ and $B_{0}=\varepsilon_{0} / \varepsilon_{p l}=\left(E_{0} / E_{p l}\right)^{4}$, we estimate the probability of the tunnelling for the compact object with the mass $M$ :

$$
D>\exp \left[-4\left(\frac{M}{m_{p l}}\right)^{3 / 4}\left(\frac{E_{p l}}{E_{0}}\right)\right] .
$$

It seems for the first sight that smaller objects collapse with bigger probability. However, this estimate does not involve the scale for de Sitter interior $E_{\text {int }}$. General constraint on the mass involving $E_{\text {int }}$ is obtained from (18). It gives $M / m_{p l}>$ $\left|B_{4}\right|>B_{0} \gamma_{\text {int }}^{4}$ where $\gamma_{\text {int }}=r_{\text {int }} / l_{p l}=\left(E_{p l} / E_{\text {int }}\right)^{2}$. Ultimately we get

$$
\frac{M}{m_{p l}}>\left(\frac{E_{0}}{E_{p l}}\right)^{4}\left(\frac{E_{p l}}{E_{\text {int }}}\right)^{8} .
$$

As we see, possibilities of formation of compact objects with the de Sitter interior depend on the scales of the interior vacuum $E_{\text {int }}$ and of the inflationary vacuum $E_{0}$. The scale of inflationary vacuum can be adopted as the GUT scale $E_{0} \simeq$ $10^{15} \mathrm{GeV}$. In the frame of the hypothesis of arising of interior de Sitter vacuum due to the phase transition at the GUT scale [41], constraint on the mass is $M>10^{11} \mathrm{~g}$. In this case only regular primordial black holes can be formed in a collapse of primordial fluctuations. In the frame of hypothesis of selfregulation of geometry near the Planck scale [39] or existence of limiting curvature of the Planck scale [40], mass range for collapsing objects admits G-lumps and RPBH including those with masses sufficiently small to evaporate and produce remnants to the end of inflation. Objects with small masses are produced with the bigger probability (20).

One more possibility for a wide range of masses is related to production of compact objects with de Sitter interior during later stages of the early Universe. The standard model of particle physics predicts a phase transition at the QCD scale of 100-200 MeV (see [77] and references therein) which can lead to a second inflationary stage with duration of about 7-10 e-foldings [78-80]. In this case the formation constraint (21) admits any mass.

We conclude that regular primordial black holes, their remnants, and G-lumps can arise in the early Universe during first and second inflationary stages, so that they can capture available particles and form graviatoms. 


\section{The Schrödinger Equation for Graviatom and Conditions of Its Existence}

The problem of a motion of a nonrelativistic charged particle in a curved spherically symmetric spacetime reduces to the Schrödinger equation [81]:

$$
-\frac{\hbar^{2}}{2 m} \Delta \psi+U(r) \psi=E \psi,
$$

where $m$ is the mass of a particle. The potential $U(r)$ includes contribution of gravity and of the DeWitt conservative selfforce acting on a charged particle with the mass $m$ and the charge $q$. It is given by [14]

$$
f_{s}=m c^{2} \frac{r_{q} r_{g}}{2 r^{3}}
$$

and directed outward the field centre. Here $r_{q}=q^{2} / m c^{2}$ is a classical electromagnetic radius of a particle. In a weak gravitational field the conservative self-force $f_{s}$ always exceeds the nonconservative radiation damping force $f_{r}=$ $2 q^{2} \ddot{v} / 3 c^{3}$, where $v$ is the particle velocity [13]. In this approximation the gravitational part of the potential is given by $m \varphi(r)=\left(m c^{2} / 2\right)\left[g_{t t}(r)-1\right]$. For a metric of the class (1) the gravitational potential reads $m \varphi=-\left(m c^{2} / 2\right)\left(\mathscr{R}_{g}(r) / r\right)$.

This approach corresponds to the Pauli approximation to the Dirac equation $[16,17]$. As we will see below, characteristic orbits of particles in the field of graviatom are in most cases located far from the characteristic Schwarzschild-de Sitter radius $r_{*}=\left(r_{\text {int }}^{2} r_{g}\right)^{1 / 3}$ which justifies application of the Pauli approximation for analysis of basic properties of graviatom. In what follows we will consider the range of frequencies $\hbar \omega<m c^{2}$ relevant for this approximation.

With taking into account the DeWitt self-force we have for the potential

$$
U(r)=\frac{m c^{2}}{2}\left(-\frac{\mathscr{R}_{g}(r)}{r}+\frac{r_{g} r_{q}}{2 r^{2}}\right)
$$

The behaviour of the potential (24) is determined by typical behaviour of the metric function $g(r)$ : in the asymptotically flat case it has not more than two zeros, one maximum at $r=0$ where $g(r)=1$ and one minimum [21]. This follows directly from the Einstein equation for the metric (1) which reads

$$
g^{\prime \prime}+\frac{2 g^{\prime}}{r}=16 \pi G p_{\perp}
$$

The pressures are given by

$$
p_{r}=-\rho, \quad p_{\perp}=-\rho-\frac{r}{2} \rho^{\prime}
$$

For the solutions satisfying the weak energy condition (nonnegative density for an observer on a timelike curve), $\rho^{\prime} \leq 0$ [21]. In the extremum of the metric function, $g^{\prime}=0$, and the type of extremum is determined by the sign of the tangential pressure $p_{\perp}$. In the asymptotically flat spacetime with de Sitter centre there is one scale of a vacuum energy, $p_{r}=p_{\perp}=$ $-\rho_{\text {int }}$ at $r=0$, and at this point $g(r)$ has a maximum. The next extremum is a minimum [21], in the region where $p_{\perp}$ is already positive (since $g^{\prime \prime}>0$ and $g^{\prime}=0$ there). In an extremum of the potential (24) the condition $U^{\prime}\left(r_{m}\right)=0$ gives

$$
g^{\prime}\left(r_{m}\right)=\frac{r_{g} r_{q}}{r_{m}^{3}}
$$

It follows that $U(r)$ has an extremum in the region where $g^{\prime}(r)>0$ and the metric function is growing. In particular, the extremum of $U(r)$ is always outside the double horizon on which $g^{\prime}=0$. The second derivative is given by, with taking into account (25) and (27),

$$
U^{\prime \prime}=\frac{m c^{2}}{2}\left(g^{\prime \prime}+\frac{3 r_{g} r_{q}}{r^{4}}\right)=16 \pi G p_{\perp}+\frac{g^{\prime}\left(r_{m}\right)}{r_{m}} .
$$

According to (27), $g^{\prime}>0$ at the extremum of $U(r)$, and also $p_{\perp}>0$ there; hence, the second derivative of the potential $U(r)$ is always positive in its extremum and the potential $U(r)$ can thus have only one minimum. For $r \rightarrow 0$, the potential $U(r)$ goes to infinity. For $r \rightarrow$ $\infty$, the metric function $g(r)$ goes asymptotically to the Schwarzschild metric, so that the potential for $r \rightarrow \infty$ goes to

$$
U(r) \longrightarrow \frac{m c^{2}}{2}\left(-\frac{r_{g}}{r}+\frac{r_{g} r_{q}}{2 r^{2}}\right),
$$

and therefore $U(r) \rightarrow-0$ as $r \rightarrow \infty$. The only minimum, $U\left(r_{m}\right)$, is thus always negative.

The radial wave function $R(r)$ in (22) satisfies the equation

$$
\frac{1}{r^{2}} \frac{d}{d r}\left(r^{2}\left(\frac{d R}{d r}\right)\right)-\frac{l(l+1)}{r^{2}} R+\frac{2 m}{\hbar^{2}}(E-U(r)) R=0 .
$$

The potential $U(r)$ can be presented as

$$
\begin{gathered}
U(r)=-\frac{G M m c^{2}}{r}\left(1-f_{g}(r)\right)+\frac{m c^{2}}{2} \frac{r_{q} r_{g}}{2 r^{2}}, \\
f_{g}(r)=\frac{8 \pi G}{r_{g} c^{2}} \int_{r}^{\infty} \rho(x) x^{2} d x<1 .
\end{gathered}
$$

Equation (30) describes a captured particle in the effective potential:

$$
U_{\text {eff }}=-\frac{G M m c^{2}}{r}\left(1-f_{g}(r)\right)+\frac{m c^{2}}{2} \frac{r_{q} r_{g}}{2 r^{2}}+\frac{\hbar^{2} l(l+1)}{2 m r^{2}} .
$$

In the Schwarzschild limit,

$$
U_{\mathrm{eff}}=-\frac{G M m c^{2}}{r}+\frac{m c^{2}}{2} \frac{r_{q} r_{g}}{2 r^{2}}+\frac{\hbar^{2} l(l+1)}{2 m r^{2}} .
$$

In this limit we see clearly that in the case of a graviatom the fine structure constant is replaced with the effective gravitational fine structure constant [13]:

$$
\alpha_{G}=\frac{G M m}{\hbar c}
$$


The corresponding Bohr radius for the graviatom with the potential $(33)$ is given by $[16,17]$

$$
r_{b}=\frac{\hbar^{2}}{G M m^{2}}=\frac{2 \hbar^{2}}{r_{g}},
$$

where $\lambda=\hbar / m c$ is the Compton wavelength of a captured particle.

The condition $r_{g}<r_{b}$ (the Bohr orbit in the R-region outside the horizon) gives

$$
r_{g}<r_{b} \longrightarrow r_{g}^{2}<2 \hbar^{2}
$$

Equation (36) gives the constraint on the mass of the graviatom nucleus:

$$
M<2 \times 10^{17}\left(\frac{m_{e}}{m}\right) \text { grams, }
$$

where $m_{e}$ is the mass of the electron.

The main condition for the existence of a graviatom is the geometrical condition:

$$
L>r_{n}+r_{p} .
$$

Here $L$ is the characteristic size of a graviatom, $r_{n}$ is the size of its nucleus, and $r_{p}$ of a captured particle. For a hydrogen-like graviatom, it reads $r_{b}>r_{n}+\lambda$.

For the metric function (2), the black hole horizon, $r_{+}=$ $2 G \mathscr{M}\left(r_{+}\right)$, satisfies $r_{+}<r_{g}$ since, for any density profile satisfying the weak energy condition $\rho^{\prime}(r) \leq 0, \mathscr{M}(r)$ is monotonically growing, and $\mathscr{M}\left(r_{+}\right)<M$. The restriction following from $r_{g}<r_{b}$ will also hold for $r_{+}$to guarantee $r_{b}>r_{+}$, so we can take $r_{n}=r_{g}$. The condition (38) in this case, $r_{b}>r_{g}+\lambda$, with taking into account (35), gives $r_{g}^{2}+r_{g} \lambda-2 \lambda^{2}=\left(r_{g}-\lambda\right)\left(r_{g}+2 \lambda\right)<0$ which follows in the constraint:

$$
r_{g}<\lambda
$$

Fulfillment of the conditions (39) guarantees fulfillment of the constraint (36).

In the case of G-lump, its characteristic size is of the order of $r_{*}[19,21]$, and the condition of the existence of the hydrogen-like graviatom reads

$$
r_{b}>r_{*}+\lambda
$$

Introducing the dimensionless quantities $x_{g}=r_{g} / r_{\text {int }}$ and $\eta=\lambda / r_{\text {int }}$, we get the inequality $x_{g}^{4 / 3}+\eta x_{g}-2 \eta^{2}<0$. With the new variable $x=x_{g}^{1 / 3}$, it reduces to $x^{4}+\eta x^{3}-2 \eta=$ $\left(x-x_{1}\right)\left(x-x_{2}\right)\left(x-x_{3}\right)\left(x-x_{4}\right)<0$. This is the 4 th order polynomial; complex conjugate roots come in pairs and make this polynomial positive, so that it can be negative only if real roots exist. The condition of their existence, $\eta \geq 0.124$, results in the following constraint:

$$
\lambda>0.124 r_{\text {int }} .
$$

This is the important constraint; only when (41) is satisfied, the geometric condition (40) holds. In this case the above polynomial has three negative and one positive root. The only possibility to satisfy (40) results in the second constraint:

$$
r_{g}<1.66 \lambda
$$

With these two constraints we estimate

$$
\frac{r_{b}}{r_{*}}>1.21
$$

For further analysis we adopt some slowly falling density profile $\rho(r)$ which should vanish for large $r$ faster than $r^{-3}$ to guarantee finiteness of the mass $M$ [21]. If we choose $\rho(r)=$ $\rho_{\text {int }}\left(r_{\text {int }} / r\right)^{4}$, we obtain

$$
f_{g}(r)=\frac{3 r_{\text {int }}^{2}}{r_{g} r} .
$$

The requirement for the Bohr orbit (35) to be outside the horizon, $r_{ \pm}<r_{g}<r_{b}$, results in

$$
f_{g}\left(r_{b}\right)<f_{g}\left(r_{g}\right), \quad \frac{3 r_{\text {int }}^{2}}{2 \hbar^{2}}<f_{g}\left(r_{g}\right)<\frac{3 r_{\text {int }}^{2}}{r_{ \pm}^{2}}
$$

where $r_{ \pm}$is the double horizon radius. It is calculated from $g\left(r_{ \pm}\right)=0, g^{\prime}\left(r_{ \pm}\right)=0$ which gives

$$
r_{ \pm}=2 G \mathscr{M}\left(r_{ \pm}\right), \quad r_{ \pm}^{2}=\frac{c^{2}}{8 \pi G \rho\left(r_{ \pm}\right)} .
$$

The first inequality in (45) gives, with taking into account (31), the constraint

$$
\frac{3}{2}\left(\frac{l_{p l}}{\lambda_{e}}\right)^{2}\left(\frac{E_{p l}}{E_{\mathrm{int}}}\right)^{4}\left(\frac{m}{m_{e}}\right)^{2}<f_{g}\left(r_{g}\right),
$$

where $m_{e}$ is the mass of the electron and $\lambda_{e}$ is its Compton wavelength.

For the GUT scale interior $E_{\mathrm{int}} \simeq 10^{15} \mathrm{GeV}$, this gives for the mass of the captured particle $m<10^{11} \mathrm{GeV}$ and for the Planck scale interior $m<m_{p l}$.

The second inequality in (45) gives, with taking into account (46), the constraint

$$
f_{g}\left(r_{g}\right)<9 \frac{\rho_{ \pm}}{\rho_{\text {int }}}=\frac{9 c^{2}}{32 \pi G^{3} M^{2}\left(r_{ \pm}\right)}<1 .
$$

It follows that the more the mass contained under the double horizon is, the better graviatom with a regular black hole can be approximated by the Schwarzschild potential (33). It suggests also that more quickly falling density profile would be more favorable for the existence of the Bohr orbits in the R-region outside the horizon.

For detailed estimates we adopt the density profile [18]:

$$
\begin{aligned}
& \rho(r)=\rho_{\mathrm{int}} e^{-r^{3} / r_{*}^{3}}, \quad r_{*}=\sqrt[3]{r_{\text {int }}^{2} r_{g}}, \\
& r_{\text {int }}^{2}=\frac{3}{8 \pi G \rho_{\text {int }}}, \quad \rho_{\text {int }}=\rho(r \longrightarrow 0),
\end{aligned}
$$


which describes vacuum polarization effects leading to de Sitter interior [39] in the semiclassical model for vacuum polarization in the gravitational field [19]. The metric function is given by

$$
g(r)=1-\frac{r_{g}}{r}\left(1-\exp \left(-\frac{r^{3}}{r_{*}^{3}}\right)\right) .
$$

A black hole exists for $r_{g} \geq r_{g(\mathrm{cr})}=1.7576 r_{\text {int }}$ [19]. The boundary value corresponds to the mass $M_{\mathrm{cr}} \simeq 10^{3}\left(E_{\mathrm{GUT}} /\right.$ $\left.E_{\text {int }}\right)^{2}$ g. With using (50) we get for the horizon $r_{+}$

$$
\frac{r_{g}}{r_{+}}\left(1-\exp \left(-\frac{r_{+}^{3}}{r_{*}^{3}}\right)\right)=1 \text {. }
$$

The most essential difference between a graviatom and the hydrogen atom is that, in the graviatom, due to the presence of the DeWitt force and modification of the Coulomb-like part of the potential, the effective potential has the minimum. This leads to an appearance of oscillatory spectrum and radiation from oscillatory levels.

With the density profile (49) the effective potential (32) takes the form

$$
\begin{aligned}
U_{\mathrm{eff}}= & -\frac{G M m c^{2}}{r}\left(1-\exp \left(-\frac{r^{3}}{r_{*}^{3}}\right)\right)+\frac{m c^{2} r_{g} r_{q}}{4 r^{2}} \\
& +\frac{\hbar^{2} l(l+1)}{2 m r^{2}} .
\end{aligned}
$$

The condition for a minimum of the function (52), $U^{\prime}\left(r_{m}\right)=$ 0 , gives the relation

$$
e^{-x}=\left(1-\frac{\tilde{r}_{q}}{r_{*} \sqrt[3]{x}}\right)(1+3 x)^{-1},
$$

where $x=r_{m}^{3} / r_{*}^{3}$ and

$$
\tilde{r}_{q}=r_{q}+\frac{2 \lambda^{2}}{r_{g}} l(l+1) .
$$

Equation (53) can be written as

$$
\frac{\tilde{r}_{q}}{r_{*}}=\left[1-(1+3 x) e^{-x}\right] \sqrt[3]{x} .
$$

For $\widetilde{r}_{q} / r_{*} \gg 1$ we have $\sqrt[3]{x}=r_{q} / \sqrt[3]{r_{\text {int }}^{2} r_{g}}$ and $r_{m}=\widetilde{r}_{q}$. For $r_{q} r_{g} \gg 2 \lambda^{2}, \widetilde{r}_{q}=r_{q}$, oscillatory levels are determined by the effective classical electromagnetic radius $r_{q}$, and the second term in (54) would provide quantum corrections to oscillatory levels.

For $\widetilde{r}_{q} / r_{*} \ll 1$ we have $x=1.9$ and

$$
r_{m}=\sqrt[3]{1.9 r_{\text {int }}^{2} r_{g}}
$$

This is the most promising case since quantum radiation which we present in the next section carries information about de Sitter interior of the graviatom nucleus.
In the general case the position of the minimum $r_{m}$ is determined by the interpolation formula:

$$
r_{m}=\widetilde{r}_{q}\left(1-\exp \left(-\frac{\tilde{r}_{q}}{\sqrt[3]{1.9 r_{\text {int }}^{2} r_{g}}}\right)\right)^{-1} .
$$

The hydrogen-like radiation dominates when the effect of the DeWitt contribution is less that of the Coulomb-like term in (33), $r_{g} r_{q} \ll \lambda^{2}$. Radiation from the oscillatory levels dominates in the opposite case, $r_{g} r_{q} \gg \lambda^{2}$.

\section{Graviatom Radiation}

4.1. Hydrogen-Like Spectrum and Radiation. The constraint (38) leads, with taking (35) into account, to $r_{b}^{3} / r_{*}^{3}>8 r_{g}^{2} / r_{\text {int }}^{2}$.

For the density profile (49) $8 r_{g(\mathrm{cr})}^{2} / r_{\text {int }}^{2} \simeq 24.7\left(E_{\text {int }} /\right.$ $\left.E_{\mathrm{GUT}}\right)^{4}$; as a result $f_{g}\left(r_{b}\right) \ll 1$ for the graviatom with a regular black hole and its remnant, and spectrum of radiation can be approximated with the high accuracy by that for the Schwarzschild potential.

In this case the energy spectrum is given by $[16,17]$

$$
E=-2 \alpha_{G}^{2} m c^{2} \frac{1}{\left[2 p+1+\sqrt{(2 l+1)^{2}+\frac{8 m A}{\hbar^{2}}}\right]^{2}},
$$

where

$$
\begin{gathered}
A=\frac{m c^{2} r_{q} r_{g}}{4}, \quad p=n-s-1, \\
s(s+1)=\frac{2 m A}{\hbar^{2}}+l(l+1),
\end{gathered}
$$

$p=0,1,2, \ldots, l \leq n, n=1,2,3, \ldots$. Here $n$ and $l$ are the principal and orbital quantum numbers, respectively. This is general formula including both the Bohr levels and oscillatory levels.

The radiation from transitions between the Bohr levels dominates when $8 m A / \hbar^{2} \ll(2 l+1)^{2}$. The condition of its domination,

$$
\left(l+\frac{1}{2}\right)^{2} \lambda^{2} \gg r_{g} r_{q}
$$

gives the constraint $M \ll 3 \times 10^{19}\left(m_{e} / m\right) \mathrm{g}$.

In the case of a black hole, the requirement $r_{g} \geq r_{g(\mathrm{cr})}$ $\left(M \geq M_{\mathrm{cr}}\right)$ results in the estimate:

$$
r_{b}^{3}>8 r_{g(\mathrm{cr})}^{2} r_{g}
$$

In the limit (60) the energy spectrum reduces to

$$
E_{B}=-\frac{m c^{2}}{2 n^{2}} \alpha_{G}^{2}
$$

In this case we have the dipole electromagnetic radiation of graviatom (dominating for the Bohr levels) similar to that 
for a hydrogen-like atom. Frequencies for the transitions $2 p \rightarrow 1 s$ and $3 p \rightarrow 1 s$ are given by

$$
\hbar \omega_{21}=\frac{3}{8} \alpha_{G}^{2} m c^{2}, \quad \hbar \omega_{31}=\frac{4}{9} \alpha_{G}^{2} m c^{2},
$$

and intensities are related by $I_{21} / I_{31}=3.161[16,17]$.

For the case of a black hole there is the natural stability condition [16, 17]: $\tau_{\mathrm{gr}}<\tau_{H}$ and $\tau_{\mathrm{gr}}<\tau_{p}$ where $\tau_{\mathrm{gr}}$ is the graviatom lifetime, $\tau_{H}$ is the black hole lifetime, and $\tau_{p}$ is the particle lifetime. The condition of indestructibility, $E_{d}<E_{b}$, where $E_{d}$ is the destructive energy (due to tidal forces, the Hawking radiation and "ionization"-eventual removing of a charged captured particle) and $E_{b}$ is the binding energy, was thoroughly studied in $[16,17]$. For the case of the hydrogenlike graviatom with the Schwarzschild potential $(f(g) \ll$ $1)$, conditions of stability and indestructibility constrain the gravitational fine structure constant within the narrow range $[16,17] 0.512<\alpha_{G}<0.625$. For this range of the coupling $\alpha_{G}$ the applied Pauli approximation holds for the frequencies of the hydrogen-like radiation (63).

In the case of G-lump the estimate (43) restricts the correction to the potential for the density profile (49),

$$
f_{g}\left(r_{b}\right)=\exp \left(-\frac{r_{b}^{3}}{r_{*}^{3}}\right)<0.17
$$

However, we cannot approximate the radiation spectrum by that for the Schwarzschild case with this accuracy since the energy is proportional to $\alpha_{G}^{2}$ and thus to $M^{2}$.

In the case of G-lump we should have to solve a separate problem with the potential (32). Preliminary consideration can be made by replacing the mass $M$ in (62) with $M(1-$ $\left.\exp \left(-r^{3} / r_{*}^{3}\right)\right)$. The rough estimate with

$$
\begin{aligned}
E_{B} & =-\frac{m c^{2}}{2 n^{2}} \alpha_{G}^{2}\left(1-2 \exp \left(-\frac{r_{b}^{3}}{r_{*}^{3}}\right)\right) \\
& =-\frac{m c^{2}}{2 n^{2}} \alpha_{G}^{2}\left(1-2 \exp \left[\left(-\frac{r_{b}^{3}}{r_{g}}\right)\left(\frac{8 \pi G \rho_{\text {int }}}{3}\right)\right]\right)
\end{aligned}
$$

gives the correction of the order of 0.34 . This estimate is relevant also for a remnant since its characteristic size, $r_{ \pm}=$ $1.414 r_{\text {int }}$, is close to $r_{*}=1.207 r_{\text {int }}$. This allows us to conclude that radiation from the Bohr levels in the case of G-lump and remnant will depend on the density of the interior de Sitter vacuum $\rho_{\text {int }}$ and would result in observational signatures for them as dark matter candidates.

\subsection{Oscillatory Spectrum and Radiation. In the case when}

$$
\left(l+\frac{1}{2}\right)^{2} \lambda^{2} \ll r_{g} r_{q}
$$

the DeWitt force is dominating, and we deal with an oscillatory spectrum and radiation.

Near the minimum, the potential reduces to

$$
U_{\mathrm{eff}}(r) \simeq U_{\mathrm{eff}}\left(r_{m}\right)+\left.\frac{1}{2} \frac{d^{2} U_{\mathrm{eff}}}{d r^{2}}\right|_{r=r_{m}}\left(r-r_{m}\right)^{2}
$$

Introducing in (30) with the effective potential (67) the new variables

$$
\chi(r)=R(r) r, \quad y=r-r_{m}
$$

we obtain the equation of a harmonic oscillator in one dimension:

$$
\frac{d^{2} \chi}{d y^{2}}+\frac{2 m}{\hbar^{2}}\left(E-\frac{m \omega^{2} y^{2}}{2}\right) \chi=0,
$$

The energy spectrum is given by

$$
\begin{array}{r}
E_{p}=-U\left(r_{m}\right)+\hbar \omega\left(p+\frac{1}{2}\right), \\
p=0,1,2, \ldots ; \quad \omega=\sqrt{\left.\frac{1}{m} \frac{d^{2} U}{d r^{2}}\right|_{r=r_{m}} .}
\end{array}
$$

The wave function being a solution to (69) has the form [82]:

$$
\chi_{n}(y)=\left(\frac{m \omega}{\pi \hbar}\right)^{1 / 4} \frac{1}{\sqrt{2^{n} n !}} \exp \left(-\frac{m \omega}{2 \hbar^{2}} y^{2}\right) H_{n}\left(y \sqrt{\frac{m \omega}{\hbar}}\right)
$$

and satisfies the normalization condition:

$$
\int_{-\infty}^{\infty} \chi_{n}^{2}(y) d y=1
$$

Matrix elements of the coordinate $y$ are

$$
y_{n, n-1}=\int_{-\infty}^{\infty} \chi_{n} \chi_{n-1} y d y=\sqrt{\frac{n \hbar}{2 m \omega}} .
$$

The intensity of an electric dipole radiation has the form [83]:

$$
P_{i f}=\frac{4 q^{2} \omega_{i f}^{4}}{3 c^{3}}\left|y_{i f}\right|^{2}
$$

where $\omega_{i f}=(1 / \hbar)\left(E_{i}-E_{f}\right)$ is the frequency of a transition $i \rightarrow$ $f$ and $y_{i f}$ are the matrix elements of the charge coordinate $y$ in the transition from the state $i$ to the state $f$.

Using (73), we obtain, for $i=n$ and $f=n-1$, the expression

$$
P_{n, n-1}=\frac{2 q^{2} \omega^{3} n \hbar}{3 m c^{3}} .
$$

For the graviatom with the de Sitter interior in the limit (66), there are two possibilities. For $r_{q} / r_{*} \gg 1$, the correction to the Coulomb part potential in (32) satisfies $f_{g}\left(r_{m}\right) \ll 3 r_{\text {int }}^{2} / \lambda^{2}$ by virtue of $r_{g} r_{q} \gg \lambda^{2}$. The graviatom can be described with the high accuracy by the Schwarzschild potential (33) which does not provide information about internal structure of the graviatom nucleus. The minimum of the potential (33) is located at $r_{m}=r_{q}$. The series in $\lambda / \sqrt{r_{q} r_{g}} \ll 1$ gives the oscillatory spectrum:

$$
E=-\frac{m c^{2} r_{g}}{4 r_{q}}+\frac{\hbar c}{r_{q}}\left(p+\frac{1}{2}\right) \sqrt{\frac{r_{g}}{2 r_{q}}}
$$

where $p=0,1,2, \ldots$ 
For a black hole as a nucleus, the condition for the minimum outside of horizon, $r_{q}>r_{g}$, leads to the constrain $M<2 \times 10^{15}\left(m_{e} / m\right)$ g. The condition (66) gives the restriction $\lambda \ll r_{q}$ which results in $Z \gg 11$ for particles captured by a black hole. Only atomic nuclei can be captured with $Z \gg$ $\sqrt{\hbar c / e^{2}}$ [13]. However, the captured nuclei with $Z \gg 11$ are quickly destroyed by the Hawking radiation from the black hole horizon. The condition for existence of such a graviatom, $k T_{H}<E_{b}$ where $E_{b}$ is a binding energy, is not satisfied for $Z \gg 11[16,17]$.

In the case of a G-lump or remnant as a graviatom nucleus, the condition (66) of capture of a particle on a level near the minimum $r_{m}=r_{q}$ leads to the constraint $r_{g} \gg$ $\lambda^{2} / r_{q}$ which excludes a nucleus with $M \leq M_{\text {cr }}$. This is not a big disaster since a spectrum in this case does not carry information about an internal structure. From this point of view, the most interesting is the case (56) when $r_{m}$ and thus frequency depend on the interior vacuum scale $\rho_{\text {int }}$.

The second possibility is specified by

$$
\frac{r_{q}}{r_{*}} \ll 1 .
$$

For the case of the density profile (49) the minimum of the effective potential (52) in the limit (66) is given by (56), and the oscillatory spectrum

$$
E=\simeq-\frac{0.425 m c^{2} r_{g}}{\left(1.9 r_{\mathrm{int}}^{2} r_{g}\right)^{1 / 3}}+\frac{0.678 \hbar c}{r_{\mathrm{int}}}\left(p+\frac{1}{2}\right), \quad p=0,1,2, \ldots,
$$

the intensity of the dipole radiation

$$
P_{n, n-1}=\frac{0.416 q^{2} n \hbar}{m r_{\mathrm{int}}^{3}},
$$

and the frequency

$$
\hbar \omega=\frac{0.678 \hbar c}{r_{\mathrm{int}}}
$$

depend on $r_{\text {int }}$ and hence on $\rho_{\text {int }}$.

The geometrical condition requires $\lambda<r_{m}$. It gives us the upper limit for the intensity

$$
P_{10}<\xi\left(\frac{l_{p l}}{r_{\text {int }}}\right)^{2} \sqrt[3]{\frac{r_{g}}{r_{\text {int }}}} \frac{c^{5}}{G}
$$

where $\xi=0.111\left(e^{2} / \hbar c\right)$ and $c^{5} / G=3.63 \cdot 10^{59} \mathrm{erg} \mathrm{s}^{-1}$. In the case of G-lump with $r_{g} \sim r_{\text {int }} \sim 10^{-25} \mathrm{~cm}$ we have $P_{10}<$ $4 \cdot 10^{40} \mathrm{erg} \mathrm{s}^{-1}$, and for a remnant and near extremal black hole $P_{10}$ is bigger. Formula (81) is valid for particles with the mass $m>\hbar / c r_{\text {int }}$ which follows from the restriction $\hbar \omega<m c^{2}$ of applicability of nonrelativistic quantum mechanics.

The energy of radiated quanta exceeds the average energy $k T_{H}=\hbar c^{3} / 8 \pi G M$ of the quantum radiation from the Schwarzschild black hole and the average energy $k T_{H}=$ $\hbar c / 4 \pi r_{\text {int }}$ from the de Sitter horizon of the interior scale $\rho_{\text {vac }}$.
The ratio of the intensity of radiated quanta in our case to the Hawking radiation of the Schwarzschild black hole is estimated as

$$
\frac{P_{10}}{P_{H(\mathrm{Sch})}} \leq 960 \times 4 \pi \times \beta \times\left(\frac{r_{g}}{r_{\text {int }}}\right)^{7 / 3} \simeq 9.68\left(\frac{r_{g}}{r_{\text {int }}}\right)^{7 / 3} .
$$

The ratio of our intensity to intensity of the Hawking radiation of the de Sitter horizon is

$$
\frac{P_{10}}{P_{H(\text { de Sitter })}} \leq 960 \pi \beta\left(\frac{r_{g}}{r_{\text {int }}}\right)^{1 / 3} \simeq 38.7\left(\frac{r_{g}}{r_{\text {int }}}\right)^{1 / 3} .
$$

4.3. Availability of Information on Graviatom Interior. The most promising case is when the frequency of oscillatory transitions (80) depends on the scale of the interior de Sitter vacuum. It could be an observational signature of a graviatom with de Sitter interior as a dark matter candidate. In this case we can estimate frequency as

$$
\hbar \omega=\frac{0.678 \hbar c}{r_{\mathrm{int}}}=0.678 \times 10^{11} \mathrm{GeV}\left(\frac{E_{\mathrm{int}}}{E_{\mathrm{GUT}}}\right)^{2} .
$$

Photons with frequencies of order $10^{11} \mathrm{GeV}$ were first observed in ultra-high-energy cosmic rays in 1962 [84]. Current experiments allow detection of photons up to $10^{11.5} \mathrm{GeV}$ (see [85] and references therein). Present observational possibilities prefer thus graviatoms with the GUT scale interior although those with the Planck scale interior can exist in principle, and probabilities of their production in a collapse are bigger, but their typical frequency, $\hbar \omega \simeq 0.7 \times 10^{19} \mathrm{GeV}$, is far from the today observational range.

The most promising case can be realized when two conditions are satisfied: the condition (66) of dominance of the oscillatory levels and the condition (77) defining appropriate location of the minimum of the effective potential.

To tell something about a graviatom nucleus, we can take roughly $r_{+}=r_{g}$ and apply the requirement that the minimum of potential must be located in the R-region outside the event horizon. The requirement $r_{g}<r_{m}$ leads for the density profile (49) to $x_{g}<1.38$. This means that the most favorite situation can take place for G-lump.

Precise estimate gives $x_{g}=1.7576, x_{m}=2.284, x_{ \pm}=$ 1.414 for a double-horizon remnant with the density profile (49). The minimum of the potential is outside of the double horizon. The Hawking temperature is zero, so that it does not disturb the oscillatory radiation of the graviatom. The case of almost extremal black hole is also possible, until $r_{m}>$ $r_{+}$, in such a case temperature is quickly going to zero, and oscillatory spectrum cannot be destroyed by the Hawking radiation.

Our predictions concerning captured particles are restricted here by the applied approach. The basic requirement of applicability of the Pauli approximation

$$
\hbar \omega<m c^{2} \longrightarrow m>\frac{\hbar}{c r_{\text {int }}}
$$


gives immediately in (84) the constraint on the mass of a captured particle $m>5 \times 10^{10} \mathrm{GeV}$ for the GUT scale of the interior de Sitter vacuum, so we can trust the above conclusions made in the frame of nonrelativistic quantum mechanics but cannot exclude that a more precise approach would open additional possibilities.

To evaluate which particle can be a captured, we apply the constraint $x_{g} \leq 1.7576$. The condition of the proper location of the minimum (77) requires $r_{q} \ll(1.7576)^{1 / 3} r_{\text {int }}$. This gives $r_{q} \ll \simeq 1.21 \times 10^{-25} \mathrm{~cm}$ for the GUT scale of the interior de Sitter vacuum. What a particle it can be? From $r_{q}=$ $e^{2} / N m_{e} c^{2}=r_{e} / N \ll 1.21 \times 10^{-25} \mathrm{~cm}$, we get $N \gg 2 \times 10^{12}$ which means $m=N m_{e} \gg 10^{9} \mathrm{GeV}$.

This range admits GUT particles with masses $10^{14} \mathrm{GeV}<$ $E_{\mathrm{GUT}}<10^{16} \mathrm{GeV}$ which could be captured by RPBH at the end of inflation. However, here the problem arises. Formation constraint restricts masses of RPBH with GUT scale interior by $M>10^{11} \mathrm{~g}$. For such a black hole, even with very quickly falling density profile (49), proper for the frequency (84), minimum (56) is located deeply inside T-region, so a particle would not be captured on an orbit but would be swallowed by a black hole. G-lumps and remnants which do not encounter this problem can be formed at the first inflationary stage only with Planck scale interior. They can capture GUT particles, but their radiation would be then in the range unavailable for near future observations.

Question arises where regular black hole remnants with GUT scale interior produced in evaporation, as well as remnants and G-lumps produced at the second inflationary stage, can capture particles with masses of the order of the GUT mass.

Speaking about RPBH produced during first inflation, one can imagine a situation when it would capture a GUT particle on some higher oscillatory level. Simple estimate $r-r_{m}>r_{g}$ with the spectrum (78) shows that it is possible, but lifetime of such a level should have to be comparable to the evaporation time $\tau \simeq\left(M / m_{p l}\right)^{3} \tau_{p l}$ [35]. In our case it gives $\tau>6.75 \times 10^{3} \mathrm{~s}$.

Such a case could be realized if some metastable level(s) with appropriate quantum number(s) $p$ would exist in the oscillatory spectrum, which cannot be excluded a priori.

Good news is that the level with energy $E_{p}>0.678 \times$ $10^{11} \mathrm{GeV}$ would not be destroyed by the Hawking radiation. Spherically symmetric regular black hole with de Sitter interior evolves during evaporation to the double-horizon remnant with zero temperature, while its maximal temperature is given by $k T_{\max }=0.2 \times 10^{11} \mathrm{GeV}[19,37,41]$.

Another channel is related to leptoquarks which can survive in galactic halos [86]. The leptoquarks arising at the GUT epoch are decayed into quarks and leptons. The quarks form nucleons in three minutes after the Big Bang. However, a part of leptoquarks survives and is accumulated in the galactic halos contributing to a dark matter [86]. The leptoquarks in the galactic halos can be captured by any of the considered here objects with de Sitter interior: by near extreme primordial black holes, by remnants, and by Glumps.

\section{Summary}

General constraint on formation of a compact object with the de Sitter interior results in the following options: at the first inflationary stage at the GUT scale, regular black holes with masses $M>10^{11} \mathrm{~g}$ can be formed with the GUT scale interior and any objects with the Planck scale interior including Glumps. There are no constraints on formation of RPBH and G-lumps during the second inflationary stage at the QCD scale.

Spectra and radiation of graviatoms are studied in the Pauli approximation to the Dirac equation. The gravitational coupling $\alpha_{G} \sim 0.5$ is much bigger than the fine structure constant $\alpha=e^{2} / \hbar c=1 / 137$. The energy levels in a graviatom are determined by $\alpha_{G}$, and relativistic correction which would allow for a particle spin is of the order of $\alpha / \alpha_{G} \ll 1$. The condition of the particle localization dimension exceeding the Compton wavelength is valid all over the paper. This means that the problem is solvable in the framework of nonrelativistic quantum mechanics, although it gives the restriction on the mass of a captured particle, $m>\hbar \omega / c^{2}$.

The electromagnetic radiation of charged particles in the field of a regular black hole exceeds the Hawking radiation from Schwarzschild black hole of the same mass. The essential difference is that a graviatom emits spectral lines which can bear information on its de Sitter interior.

Hydrogen-like spectrum and radiation of graviatom with $\mathrm{RPBH}$ as a nucleus is described with the high accuracy by those for the Schwarzschild black hole. Requirements of stability of graviatom with respect to tidal forces and Hawking radiation constraint the gravitational coupling within the narrow range $0.512<\alpha_{G}<0.625$. Dominating is the dipole radiation which does not carry information about de Sitter interior of graviatom. In the case of G-lump and RPBH remnant, approximation of the potential by the corrected Schwarzschild potential suggests that hydrogenlike radiation will depend on the density of the interior de Sitter vacuum $\rho_{\text {int }}$ and result in observational signatures for G-lump and remnant as dark matter candidates. This case needs further detailed investigation.

The oscillatory radiation can depend essentially on the interior vacuum scale and gets in the range of ultrahigh cosmic ray energies.

Typical features of graviatoms radiation can result in observational signatures for G-lumps and $\mathrm{RPBH}$ remnants as dark matter candidates, as well as provide information about their interior de Sitter vacuum.

Let us note that regular primordial black holes, their remnants, and G-lumps can also serve as a very sensitive universal probe for a scale of inhomogeneities in the very early Universe being in this context the most elusive among dark matter candidates [87].

\section{Appendix}

\section{Characteristic Length Scales of Graviatoms}

Graviatoms have rich internal structure, characterized by several essential length scales which we summarize below. 
Characteristic size of a graviatom $L$ satisfies the geometrical condition $L>r_{n}+r_{p}$ where $r_{n}$ is the size of the nucleus and $r_{p}$ is the size of a captured particle.

The characteristic length of any de Sitter-Schwarzschild spacetime $r_{*}=\left(r_{\text {int }}^{2} r_{g}\right)^{1 / 3}$ comes as the radius of the direct matching of the de Sitter and Schwarzschild metrics, 1 $r_{*}^{2} / r_{\text {int }}^{2}=1-r_{g} / r_{*}$. It depends on both gravitational radius $r_{g}=2 G M c^{-2}$ and characteristic scale of the interior de Sitter vacuum $r_{\text {int }}$ related to its density by $r_{\text {int }}^{2}=3 c^{2} / 8 \pi G \rho_{\text {int }}$. For the GUT scale $\simeq 10^{15} \mathrm{GeV}$ interior, $r_{\text {int }} \simeq 2.4 \times 10^{-25} \mathrm{~cm}$. For the Planck scale, $r_{\text {int }}=r_{p l}$. Characteristic scale $r_{*}$ gives rough estimate for the size of a G-lump. More precise estimate involves critical gravitational radius $r_{g(\mathrm{cr})}$, related to critical mass $M_{\mathrm{cr}}$, actually mass of the remnant which gives the boundary between black holes $\left(M>M_{\mathrm{cr}}\right)$ and G-lumps $\left(M<M_{\mathrm{cr}}\right)$. It is given by $M_{\mathrm{cr}}=\beta m_{p l} \sqrt{\rho_{p l} / \rho_{\text {int }}}$ where the numerical coefficient $\beta$ depends on the model. For the density profile (49), $M_{\mathrm{cr}} \simeq 0.3 m_{p l} \sqrt{\rho_{p l} / \rho_{\mathrm{int}}}$ and $r_{g(\mathrm{cr})}=1.7576 r_{\mathrm{int}}$. In this case, $r_{*}>0.69 r_{g}$ for a G-lump, and $r_{*}<0.69 r_{g}$ for a black hole.

The black hole horizon $r_{+}=2 G M\left(r_{+}\right)$satisfies always $r_{+}<r_{g}$. Double horizon $r_{ \pm}=2 G \mathscr{M}\left(r_{ \pm}\right)$gives roughly a size of RBH remnant. Masses of remnants $\left(r_{g}=r_{g(\mathrm{cr})}\right)$ range from $m_{p l}$ for the Planck scale interior, through $\sim 10^{3} \mathrm{~g}$ for the GUT scale interior, up to $\sim 10^{32} \mathrm{~g}\left(r_{g} \sim 10^{4} \mathrm{~cm}\right)$ for the QCD scale interior. Regular remnants are stable, and their population includes all remnants of RPBH evaporated till now.

The gravitational analogue of the Bohr radius is given by $r_{b}=\hbar^{2} / G M m^{2}=2 \lambda^{2} / r_{g}$, where $\lambda=\hbar / m c$ is the Compton wavelength of a captured particle. The hydrogenlike radiation dominates in the case when $r_{g} r_{q} \ll \lambda^{2}$, where $r_{q}=q^{2} / m c^{2}$ is electromagnetic radius of a captured particle.

In the case $r_{g} r_{q} \gg \lambda^{2}$, the oscillatory radiation is dominating. Minimum of the graviatom potential $r_{m}$, responsible for its existence, is always outside the double horizon. For the case $r_{q} \gg r_{*}$, oscillatory levels are determined by $r_{q}$, with quantum corrections depending on $r_{g}$; in this case, $r_{m}=$ $r_{q}+2 \lambda^{2} l(l+1) / r_{g}$. In the most promising for the observational manifestations case, when $r_{q} \ll r_{*}$, location of the minimum $r_{m}$ depends directly on $r_{\text {int }}$ and thus on the interior vacuum density, $r_{m}=\sqrt[3]{1.9 r_{\text {int }}^{2} r_{g}}$ for the density profile (49). For a remnant with the density profile (49), $r_{g}=1.7576 r_{\text {int }}, r_{m}=$ $2.284 r_{\text {int }}, r_{ \pm}=1.414 r_{\text {int }}$, and $r_{*}=1.207 r_{\text {int }}$.

\section{Acknowledgments}

This work was supported by the Polish Ministry of Science and Education for the research project "Globally Regular Configurations in General Relativity Including Classical and Quantum Cosmological Models, Black Holes and ParticleLike Structures (solitons)" in the frame of the "Polish-Russian Agreement for collaboration in the Field of Science and Technology" and by the Polish National Science Center through the Grant 5828/B/H03/2011/40.

\section{References}

[1] S. Blinnikov and M. Y. Khlopov, "On possible effects of mirror particles," Soviet Journal of Nuclear Physics, vol. 36, p. 472, 1982.

[2] S. Blinnikov and M. Y. Khlopov, "Possible astronomical effects of mirror particles," Soviet Astronomy, vol. 27, pp. 371-375, 1983.

[3] M. Y. Khlopov, G. Beskin, N. Bochkarev, L. Pustylnik, and S. Pustylnik, "Observational physics of mirror Word," Soviet Astronomy, vol. 35, p. 21, 1991.

[4] S. Hawking, "Gravitationally collapsed objects of very low mass," Monthly Notices of the Royal Astronomical Society, vol. 152 , p. $75,1971$.

[5] C. W. Misner, "Relativistic fluids in cosmology," in Batelle Recontres in Mathematics and Physics, M. de Witt and J. Wheeler, Eds., p. 117, 1967.

[6] V. V. Flambaum and J. C. Berengut, "Atom made from charged elementary black hole," Physical Review D, vol. 63, no. 8, Article ID 084010, 2001.

[7] E. G. Floratos, G. K. Leontaris, and N. D. Vlachos, "Gravitational atom in compactified extra dimensions," Physics Letters $B$, vol. 694, no. 4-5, pp. 410-416, 2011.

[8] A. B. Gaina and F. G. Kochorbé, "S matrix and resonance states in the Kerr-Newman geometry," Journal of Experimental and Theoretical Physics, vol. 65, pp. 211-215, 1987.

[9] I. M. Ternov and A. B. Gaina, "Energy spectrum of the Dirac equation for the Scharzschild and Kerr fields," Soviet Physics Journal, vol. 31, no. 2, pp. 157-163, 1988.

[10] A. B. Gaina and O. B. Zaslavskii, "On quasilevels in the gravitational field of a black hole," Classical and Quantum Gravity, vol. 9, no. 3, pp. 667-676, 1992.

[11] A. Lasenby, C. Doran, J. Pritchard, A. Caceres, and S. Dolan, "Bound states and decay times of fermions in a Schwarzschild black hole background," Physical Review D, vol. 72, no. 10, Article ID 105014, 2005.

[12] M. S. Pindzola, "Inelastic scattering of electrons by black hole atoms," Journal of Physics B, vol. 42, Article ID 095202, 2009.

[13] M. L. Fil'chenkov, "Quantum radiation of a charged particle in a Schwarzschild field," Astronomische Nachrichten, vol. 311, pp. 223-226, 1990.

[14] C. M. DeWitt and B. S. DeWitt, "Falling charges," Physica, vol. 1, p. 3, 1964.

[15] M. L. Fil'chenkov, "Behavior of a charged particle in a Schwarzschild field," Izvestiya Vysshikh Uchebnykh Zavedenii Fizika, no. 7, pp. 75-82, 1998.

[16] Y. P. Laptev and M. L. Filchenkov, "Electromagnetic and gravitational radiation of graviatoms," Astronomical and Astrophysical Transactions, vol. 25, pp. 33-42, 2006.

[17] M. L. Filchenkov and Y. P. Laptev, "Graviatom dipole radiation," Gravitation and Cosmology, vol. 12, pp. 65-68, 2006.

[18] I. G. Dymnikova, "Vacuum nonsingular black hole," General Relativity and Gravitation, vol. 24, pp. 232-242, 1992.

[19] I. G. Dymnikova, "De Sitter-Schwarzschild black hole: its particlelike core and thermodynamical properties," International Journal of Modern Physics D, vol. 5, no. 5, pp. 529-540, 1996.

[20] I. Dymnikova and M. Korpusik, "Regular black hole remnants in de Sitter space," Physics Letters B, vol. 685, no. 1, pp. 12-18, 2010.

[21] I. Dymnikova, "The cosmological term as a source of mass," Classical and Quantum Gravity, vol. 19, no. 4, pp. 725-739, 2002.

[22] J. H. MacGibbon, "Can Planck-mass relics of evaporating black holes close the Universe?” Nature, vol. 329, pp. 308-309, 1987. 
[23] K. Rajagopal, M. S. Turner, and F. Wilczek, "Cosmological implications of axinos," Nuclear Physics B, vol. 358, no. 2, pp. 447-470, 1991.

[24] B. J. Carr, J. H. Gilbert, and J. E. Lidsey, "Black hole relics and inflation: limits on blue perturbation spectra," Physical Review D, vol. 50, no. 8, pp. 4853-4867, 1994.

[25] R. J. Adler, P. Chen, and D. I. Santiago, “The generalized uncertainty principle and black hole remnants," General Relativity and Gravitation, vol. 33, no. 12, pp. 2101-2108, 2001.

[26] P. Chen and R. J. Adler, "Black hole remnants and dark matter," Nuclear Physics B, vol. 124, pp. 103-106, 2003.

[27] E. Bugaev and P. Klimai, "Constraints on amplitudes of curvature perturbations from primordial black holes," Physical Review D, vol. 79, no. 10, Article ID 103511, 2009.

[28] M. Kesden and S. Hanasoge, "Transient solar oscillations driven by primordial black holes," Physical Review Letters, vol. 107, no. 11, Article ID 111101, 2011.

[29] F. S. N. Lobo, G. J. Olmo, and D. Rubiera-Farcia, "Semiclassical geons as solitonic black hole remnants," Journal of Cosmology and Astroparticle Physics, vol. 2013, 2013.

[30] B. J. Carr, "Primordial black holes-recent developments," ECONFCO41213, 0204, 2004, http://arxiv.org/abs/astro-ph/ 0504034 .

[31] B. J. Carr, "Primordial black holes as a probe of cosmology and high energy physics," Lecture Notes in Physics, vol. 631, pp. 301321, 2003.

[32] M. Y. Khlopov and S. G. Rubin, Cosmological Pattern of Microphysics in Inflationary Universe, Kluwer Academic, Dordrecht, The Netherlands, 2004.

[33] A. M. Green and A. R. Liddle, "Constraints on the density perturbation spectrum from primordial black holes," Physical Review D, vol. 56, no. 10, pp. 6166-6174, 1997.

[34] M. Drees and E. Erfani, "Primordial black holes in singlefield inflation models," Journal of Cosmology and Astroparticle Physics, vol. 1201, article 035, 2012.

[35] B. J. Carr, K. Kohri, Y. Sendouda, and J. Yokoyama, "New cosmological constraints on primordial black holes," Physical Review D, vol. 81, no. 10, Article ID 104019, 2010.

[36] L. Susskind, “The world as a hologram," Journal of Mathematical Physics, vol. 36, no. 11, pp. 6377-6399, 1995.

[37] I. Dymnikova, "Regular black hole remnants," in Proceedings of the Invisible Universe International Conference, Paris, France, June-July 2009, AIP: New York, NY, USA, 50, 2010.

[38] E. B. Gliner, "Algebraic properties of the energy-momentum tensor and vacuum-like states of matter," Journal of Experimental and Theoretical Physics, vol. 22, p. 378, 1966.

[39] E. Poisson and W. Israel, "Structure of the black hole nucleus," Classical and Quantum Gravity, vol. 5, no. 12, pp. L201-L205, 1988.

[40] V. P. Frolov, M. A. Markov, and V. F. Mukhanov, "Black holes as possible sources of closed and semiclosed worlds," Physical Review D, vol. 41, no. 2, pp. 383-394, 1990.

[41] I. Dymnikova, "Internal structure of nonsingular spherical black holes," in Internal Sructure of Black Holes and Spacetime Singularities, M. Burko and A. Ori, Eds., p. 422, Bristol In-t of Physics and the Israel Physical Society, 1997.

[42] A. Perez, "Spin foam models for quantum gravity," Classical and Quantum Gravity, vol. 20, no. 6, pp. R43-R104, 2003.

[43] C. Rovelli, Quantum Gravity, Cambridge University Press, Cambridge, UK, 2004.
[44] A. Bonanno and M. Reuter, "Renormalization group improved black hole spacetimes," Physical Review D, vol. 62, Article ID 043008, 21 pages, 2000.

[45] P. Nicolini, "Noncommutative black holes, the final appeal to quantum gravity: a review," International Journal of Modern Physics A, vol. 24, no. 7, pp. 1229-1308, 2009.

[46] P. Nicolini, A. Smailagic, and E. Spallucci, "Noncommutative geometry inspired Schwarzschild black hole," Physics Letters B, vol. 632, no. 4, pp. 547-551, 2006.

[47] R. B. Mann and P. Nicolini, "Cosmological production of noncommutative black holes," Physical Review D, vol. 84, Article ID 064014, 13 pages, 2011.

[48] I. G. Dymnikova, "The algebraic structure of a cosmological term in spherically symmetric solutions," Physics Letters B, vol. 472, no. 1-2, pp. 33-38, 2000.

[49] I. Dymnikova, "Spherically symmetric space-time with regular de Sitter center," International Journal of Modern Physics D, vol. 12, no. 6, pp. 1015-1034, 2003.

[50] I. Dymnikova and E. Galaktionov, "Vacuum dark fluid," Physics Letters B, vol. 645, no. 4, pp. 358-364, 2007.

[51] I. Dymnikova and E. Galaktionov, "Dark ingredients in one drop," Central European Journal of Physics, vol. 9, no. 3, pp. 644653, 2011.

[52] I. Dymnikova and B. Sołtysek, "Spherically symmetric spacetime with two cosmological constants," General Relativity and Gravitation, vol. 30, pp. 1775-1793, 1998.

[53] I. Dymnikova and B. Sołtysek, "Nonsingular cosmological black hole," in Particles, Fields and Gravitation, J. Rembielinsky, Ed., p. 460, AIP, New York, NY, USA, 1998.

[54] I. Dymnikova, "Cosmological term, mass, and space-time symmetry," in Beyond the Desert: Proceedings of the 4th Tegernsee International Conference on Particle Physics Beyond the Standard Model, Germany, June 2003, H. V. Klapdor-Kleinhaus, Ed., p. 485, Springer, Berlin, Germany, 2004.

[55] I. Dymnikova, "Spacetime symmetry and mass of a lepton," Journal of Physics A, vol. 41, Article ID 304033, 2008.

[56] A. Bonanno and M. Reuter, "Spacetime structure of an evaporating black hole in quantum gravity," Physical Review D, vol. 73, no. 8, Article ID 083005, 2006.

[57] Y. S. Myung, Y.-W. Kim, and Y.-J. Park, "Quantum cooling evaporation process in regular black holes," Physics Letters B, vol. 656, no. 4-5, pp. 221-225, 2007.

[58] M. Y. Khlopov, "Primordial black holes," Research in Astronomy and Astrophysics, vol. 10, no. 6, p. 495, 2010.

[59] Y. B. Zeldovich and I. D. Novikov, Soviet Astronomy, vol. 10, p. $602,1967$.

[60] V. A. Kuzmin and I. I. Tkachev, "Ultrahigh-energy cosmic rays, superheavy long-lived particles, and matter creation after inflation," JETP Letters, vol. 68, pp. 271-275, 1998.

[61] V. A. Kuzmin and I. I. Tkachev, "Ultra-high-energy cosmic rays and inflation relics," Physics Reports, vol. 320, pp. 199-221, 1999.

[62] V. A. Kuzmin and I. I. Tkachev, "Matter creation via vacuum fluctuations in the early Universe and observed ultrahigh energy cosmic ray events," Physical Review D, vol. 59, Article ID 123006, 8 pages, 1999.

[63] L. Hui and E. D. Stewart, "Superheavy dark matter from thermal inflation,” Physical Review D, vol. 60, Article ID 023518, 6 pages, 1999.

[64] A. E. Faraggi, K. A. Olive, and M. Prospelov, "Probing the desert with ultra-energetic neutrinos from the sun and the earth," Astroparticle Physics, vol. 13, pp. 31-43, 2000. 
[65] A. A. Grib and V. Y. Dorofeev, "Creation of particles and entropy in the early friedmann universe," International Journal of Modern Physics D, vol. 3, p. 731, 1994.

[66] I. F. M. Albuquerque, L. Hui, and E. W. Kolb, "High energy neutrinos from superheavy dark matter annihilation," Physical Review D, vol. 64, Article ID 083504, 10 pages, 2001.

[67] I. Dymnikova and M. Krawczyk, "First post-inflationary particles equation of state," in Birth of the Universe, F. Occhionero, Ed., Springer, New York, NY, USA, 1994.

[68] I. Dymnikova and M. Krawczyk, "Equation of state and temperature of massive nonrelativistic bosons arising in the universe at the first stage of reheating," Modern Physics Letters A, vol. 10, p. 3069, 1995.

[69] S. Capozziello, R. de Ritis, I. Dymnikova, C. Rubano, and P. Scudellaro, "Inflationary cosmology without slow rolling," Physics Letters A, vol. 203, no. 1, pp. 18-22, 1995.

[70] S. Capozziello, R. de Ritis, I. Dymnikova, C. Rubano, and P. Scudellaro, "Temperature at the first stage ofreheating in in flationary cosmology without slow rolling," Nuovo Cimento B, vol. 111, pp. 623-630, 1996.

[71] I. Dymnikova and M. Khlopov, "Decay of cosmological constant as bose condensate evaporation," Modern Physics Letters A, vol. 15, no. 38-39, pp. 2305-2314, 2000.

[72] B. K. Harrison, K. S. Thorne, M. Wakano, and J. A. Wheeler, Gravitational Theory and Gravitational Collapse, University of Chicago Press, 1965.

[73] A. V. Vilenkin, "Quantum creation of universes," Physical Review D, vol. 30, no. 2, pp. 509-511, 1984.

[74] M. L. Fil'chenkov, "The pre-de Sitter universe in terms of quantum mechanics," Physics Letters B, vol. 354, no. 3-4, pp. 208-212, 1995.

[75] M. L. Fil'chenkov, "Quantum collapse and the birth of a new universe," Physics Letters B, vol. 441, pp. 34-39, 1998.

[76] E. Kamke, Differentialgleichungen. Lösungmethoden und Lösungen. I. Gewöhnliche Differentialgleichungen, Chelsea Publishing Company, Leipzig, Germany, 1959.

[77] D. Boyanovsky, H. J. de Vega, and D. J. Schwarz, "Phase transitions in the early and present universe," Annual Review of Nuclear and Particle Science, vol. 56, pp. 441-500, 2006.

[78] T. Boeckel and J. Schaffner-Bielich, "A little inflation in the early universe at the QCD phase transition," Physical Review Letters, vol. 105, Article ID 041301, 2010.

[79] D. H. Lyth and E. D. Stewart, "Cosmology with a TeV mass Higgs field breaking the grand-unified-theory gauge symmetry," Physical Review Letters, vol. 75, no. 2, pp. 201-204, 1995.

[80] N. Borghini, W. N. Cottingham, and R. V. Mau, "Possible cosmological implications of the quark-hadron phase transition," Journal of Physics G, vol. 26, p. 771, 2000.

[81] K. Kuchař, "Gravitation, geometry, and nonrelativistic quantum theory," Physical Review D, vol. 22, no. 6, pp. 1285-1299, 1980.

[82] L. D. Landau and E. M. Lifshitz, Quantum Mechanics, Fizmatgiz, Moscow, Russia, 1963.

[83] H. A. Bethe and E. E. Salpeter, Quantum Mechanics of One- and Two-Electron Atoms, Springer, Berlin, Germany, 1957.

[84] J. Linsley, "Evidence for a primary cosmic-ray particle with energy 1020 eV," Physical Review Letters, vol. 10, no. 4, pp. 146$148,1963$.

[85] O. E. Kalashev, G. I. Rubtsov, and S. V. Troitsky, "Sensitivity of cosmic-ray experiments to ultrahigh-energy photons: reconstruction of the spectrum and limits on the superheavy dark matter," Physical Review D, vol. 80, no. 10, Article ID 103006, 2009.

[86] A. A. Grib and Y. V. Pavlov, "Do active galactic nuclei convert dark matter into visible particles?" Modern Physics Letters A, vol. 23, no. 16, pp. 1151-1159, 2008.

[87] I. Dymnikova and M. Khlopov, "Regular black hole remnants with de Sitter interior as a universal probe of inhomogeneity of early Universe," In press. 

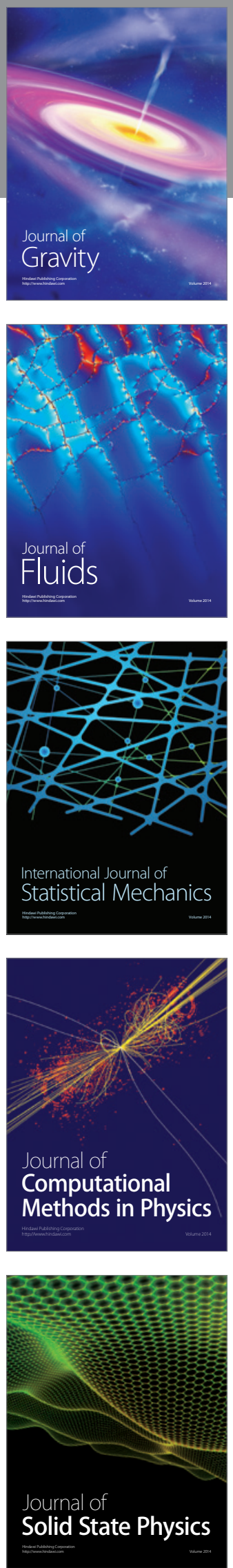

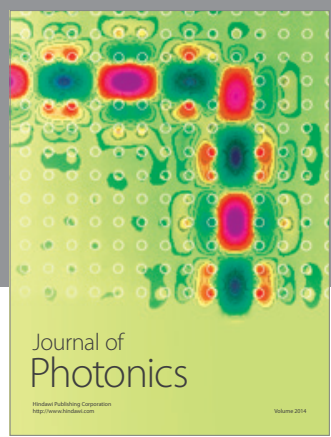

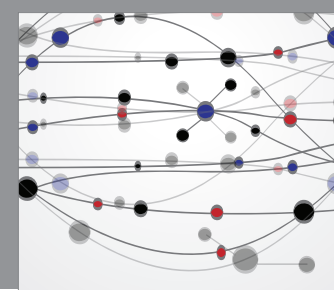

The Scientific World Journal

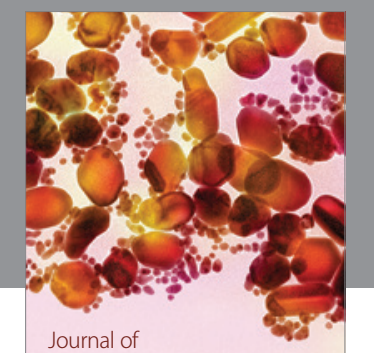

Soft Matter
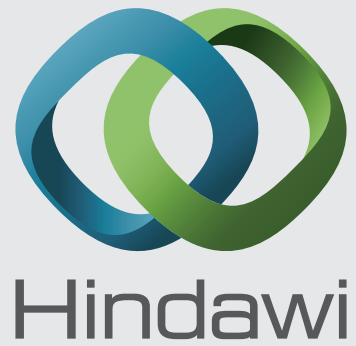

Submit your manuscripts at

http://www.hindawi.com
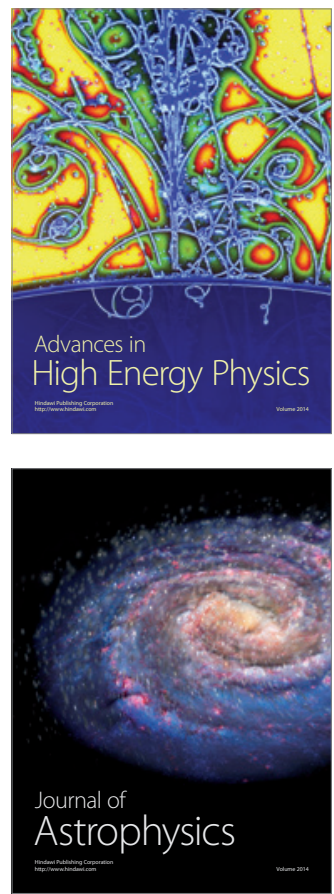
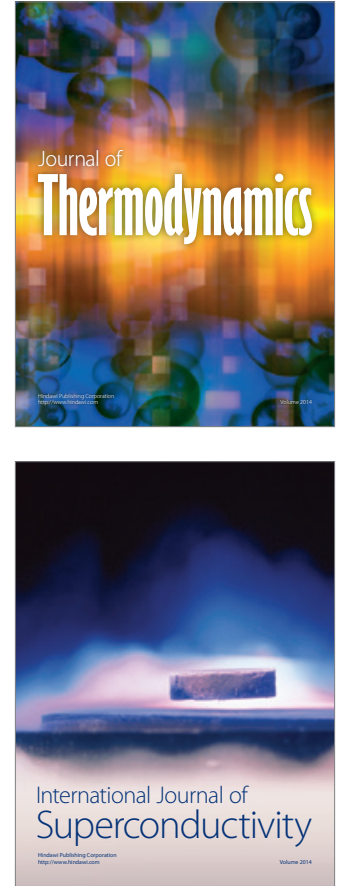
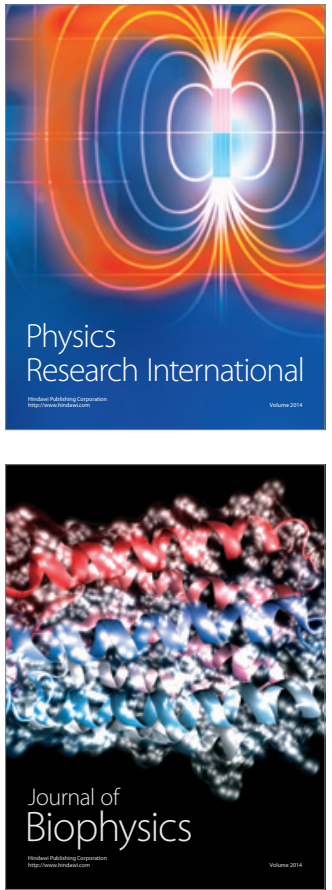
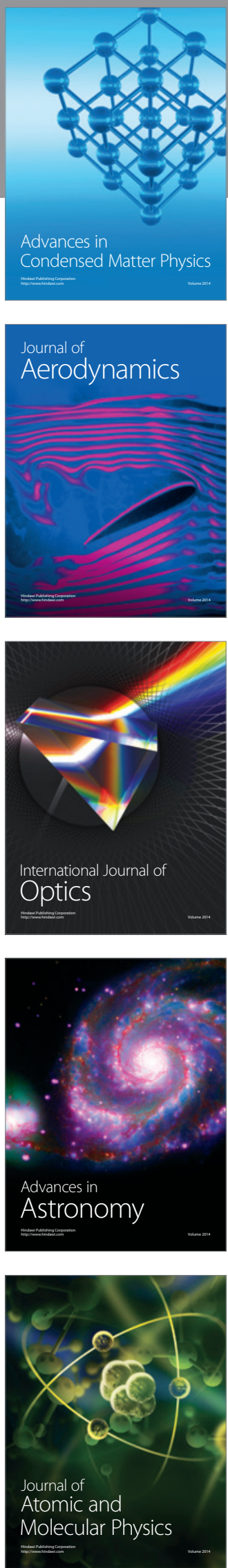